\title{
Práctica profesional penitenciaria en la Universidad Nacional Experimental de la Seguridad de Venezuela durante el periodo 2015 - 2017
}

\section{Professional penitentiary practice at the National Experimental University of Security of Venezuela during the period 2015 - 2017}

\author{
Roger José López Rodríguez \\ rogerlopezunes@gmail.com \\ Universidad Nacional Experimental de la Seguridad - Venezuela \\ Julián Ojeda Bastidas \\ julianojeda4488@gmail.com \\ Universidad Nacional Experimental de la Seguridad - Venezuela
}

Recibido: 16-10-2016 / Revisado: 23-10-2016 / Aceptado: 13-11-2016 / Publicado: 10-04-2017

\begin{abstract}
RESUMEN
Este trabajo de investigación tiene como objetivo analizar la unidad curricular la práctica profesional penitenciaria como eje transversal de la estructura curricular en el fortalecimiento del proceso de enseñanzaaprendizaje en el programa nacional de formación de la licenciatura en servicios penitenciarios en la Universidad Nacional Experimental de la Seguridad de Venezuela, el interés de realizar esta investigación nace en virtud que, de la importancia de las prácticas profesionales en el proceso formación continua, lo cual constituye la principal herramienta para asegurar y garantizar el desempeño del rol del servidor penitenciario. Los basamentos teóricos son de Delgado (2012), "estar en contacto con la institución educativa desde el inicio de la formación profesional, implica alcanzar un proceso de socialización que muestra lo complejo de la realidad socio-educativa" (pág. 48). Metodológicamente es una investigación de campo, se aplicó una encuesta a una muestra de quince (180) sujetos de investigación. El análisis de resultados permitió constatar que la muestra seleccionada reconoce la importancia de las prácticas profesionales penitenciaras. Sin embargo, es necesaria la continua las mejoras y actualizaciones de los contenidos programáticos.
\end{abstract}

Palabras clave: Formación, Servicio Penitenciario, Prácticas Profesionales
The aim of this research work is to analyze the curricular unit of the prison professional practice as a transversal axis of the curricular structure in the strengthening of the teaching- learning process in the national program of formation of the penitentiary services degree at the National Experimental University of the Security of Venezuela, the interest in carrying out this research is based on virtue of the importance of professional practices in the ongoing training process, which is the main tool to ensure and guarantee the performance of the prison server role. The theoretical foundations are Delgado (2012), "to be in contact with the educational institution since the beginning of professional training, involves achieving a process of socialization that shows the complex socio-educational reality" (page 48). Methodologically it is a field investigation, a survey was applied to a sample of fifteen (180) research subjects. The analysis of results allowed to verify that the selected sample recognizes the importance of professional penitentiary practices. However, it is necessary to continue the improvements and updates of the programmatic contents.

Key words: Training, Penitentiary Service, Professional Practices 


\section{INTRODUCCIÓN}

El Estado Venezolano a partir del año 1999, producto del nuevo proyecto de país plasmado en la Constitución de la República Bolivariana de Venezuela, implementa cambios significativos en el ordenamiento jurídico ampliando los derechos y garantías especialmente a los grupos más vulnerables, entre ellos los privados y las privadas de libertad al consagrar el régimen abierto como uno de los pilares del nuevo penitenciarismo en el artículo 272, y, en la misma línea coincidiendo con la reforma del Código Orgánico Procesal Penal desde 1999. Sin embargo, en Venezuela los cuerpos de seguridad del Estado, se ven sometidos a múltiples presiones sociales, entre ellas el reclamo de la violación de derechos humanos, el hacinamiento de los establecimientos penitenciarios producto de los retardos procesales. Esta realidad compleja que caracteriza al sistema penitenciario es producto fundamentalmente, de la marcada tendencia que ha prevalecido en el país a la aplicación de la pena privativa de libertad, medida que no aborda las causas que originan el problema del delito y genera una alta tasa de privados de libertad en situación de procesados, estatus jurídico que no permite la aplicación de medidas alternativas a la pena, hecho contrario al nuevo enfoque constitucional del sistema penitenciario.

Entre los cambios desarrollados por el Estado venezolano, está la creación de las instituciones de educación universitaria especializadas en seguridad. En este contexto, nace la Universidad Nacional Experimental de la Seguridad, la cual contempla entre sus programas de formación el Programa Nacional de Formación de Licenciatura en Servicios Penitenciarios, con lo cual se inicia el proceso de transformación de la concepción del modelo educativo para la formación de los funcionarios de seguridad ciudadana y en especial, en servicios penitenciarios con el propósito de formar al nuevo funcionario de la seguridad con un importante componente
Seguridad (UNES), institución educativa destinada a la formación de los funcionarios y las funcionarias de los cuerpos de seguridad que ha incorporado dentro de su pensum de estudios, la práctica profesional penitenciaria, en aras de dar cumplimiento al mandato constitucional de la formación académica integral del profesional en el área penitenciaria en Venezuela, dado el rol que juega y la responsabilidad que asume en el proceso de reinserción social del privado de libertad.

En tal sentido, con propósito de contribuir con la vinculación de la teoría y su aplicación en el ejercicio práctico en la formación del profesional penitenciario, facilitar el desarrollo de habilidades para identificar y ejecutar acciones necesarias en la solución de los problemas; generar conocimiento permanente de la realidad penitenciaria, fortalecería la vinculación social del discente de la Universidad Nacional Experimental de la Seguridad con el sistema penitenciario, es incorporada la unidad curricular Práctica Profesional Penitenciaria en el diseño curricular de la Licenciatura en Servicios Entre los integrantes del sistema de seguridad ciudadana, está el sistema penitenciario, último eslabón en la cadena de la política criminal venezolana, tiene su fundamento jurídico en el artículo 292 de la Constitución de la República Bolivariana de Venezuela de 1999, en el en el currículo básico por el respeto de los derechos humanos.

En los últimos años existe una marcada tendencia desde el Estado por la consolidación de una cultura de paz y la transformación de la función policial lo cual se evidencia en la creación Universidad Nacional Experimental de la Penitenciarios se estableció en el año 2015 mediante el acuerdo $\mathrm{N}^{\circ} 000082$ mediante el cual dicta el Reglamento de Prácticas Profesionales de la Universidad Nacional Experimental de la Seguridad. 
En virtud de lo anterior, surge la inquietud de analizar la unidad curricular, la práctica profesional penitenciaria como eje transversal de la estructura curricular en el fortalecimiento del proceso de enseñanzaaprendizaje en el Programa Nacional de Formación de la Licenciatura en Servicios Penitenciarios en la Universidad Nacional Experimental de la Seguridad de Venezuela.

Las políticas públicas desarrolladas por los órganos que conforman el sistema de seguridad ciudadana del Estado Venezolano están fundamentadas en la noción de Estado social de derecho, justicia y de paz. Principios que se sustentan en la Constitución de la República Bolivariana de Venezuela de 1999. Estas se materializan con respecto a la garantía de los derechos humanos, así como, el desarrollo de acciones para la prevención del delito y reinserción social del privado de libertad. En la cual el Estado venezolano reconoce en forma expresa que, "garantizará un sistema penitenciario que asegure la rehabilitación del interno o interna y el respeto a sus derechos humanos". Este enfoque constitucional del sistema penitenciario plasmado en el artículo 292se orienta a la aplicación preferente de las "fórmulas de cumplimiento de penas no privativas de la libertad a las medidas de naturaleza reclusoria".

Dentro de este marco normativo, el artículo 292 contempla la creación de un ente penitenciario con carácter autónomo y con personal exclusivamente técnico, bajo la dirección de penitenciaritas aplicación de medidas privativas de libertad, medida que no aborda las causas que originan el problema del delito y genera una alta tasa de privados de libertad, demanda de la formación académica especializada e integral de profesionales con credenciales académicas universitarias, como respuesta a este mandato constitucional, el Estado, por una parte, creó el Ministerio del Poder Popular para el
Servicio Penitenciario. Sin duda, el sistema penitenciario venezolano y su dinámica social carcelaria dada la tendencia en Venezuela hacia la profesionales. De allí, la importancia que se le da a la formación académica integral del profesional en el área penitenciaria en Venezuela, dado el rol que juega y la responsabilidad que asume en el proceso de reinserción social del privado de libertad y, su transformación en el hombre nuevo materializado en ciudadanas y ciudadanos respetuosos de las leyes y amantes de la vida y la unión familiar.

Dentro de esta perspectiva, la Práctica Profesional constituye una herramienta vital en el proceso de formación del profesional en servicios penitenciarios. Esta unidad curricular comprende un conjunto de actividades académicas conducentes a la formación del estudiante universitario en diferentes disciplinas profesionales. Representa una técnica educativa que vincula la teoría con la práctica, el aprendizaje con el trabajo, la enseñanza intelectual con el quehacer operacional laboral. La formación del profesional en el ámbito requiere instruir, educar, orientar, con prácticas profesionales penitenciarias como oportunidad formativa para el desarrollo en el espacio laboral.

Cabe destacar que, en la profesionalización de las o los estudiantes en área de conocimiento es necesario recibir, durante la formación académica, basamentos teóricos, así como, disponer de herramientas e instrumentos que coadyuven al desarrollo de técnicas y habilidades trasmitidas en la actividad universitaria práctica, los resultados derivarían en la ejecución de competencias en la ocupación profesional o actividad laboral.

En este contexto, con el propósito de contribuir con la vinculación de la teoría y su aplicación en el ejercicio práctico de la formación del profesional penitenciario, facilitar el desarrollo de habilidades para identificar y ejecutar acciones necesarias en la 
solución de los problemas; generar conocimiento permanente de la realidad penitenciaria, fortalecería la vinculación social del docente de la Universidad Nacional Experimental de la Seguridad con el sistema penitenciario. La unidad curricular Práctica Profesional Penitenciaria en el diseño curricular de la Licenciatura en Servicios Penitenciarios se estableció en el año 2015 mediante el acuerdo $\mathrm{N}^{\circ} 000082$ mediante el cual dicta el Reglamento de Prácticas Profesionales de la Universidad Nacional Experimental de la Seguridad.

Autores como, Delgado (2012), plantea que la práctica en espacios reales que permiten la apropiación de la realidad y el contraste teórico - metodológico al parecer es insuficiente y, en consecuencia, el proceso de reflexión - actuación en la cotidianidad de lo escolar escasamente se demuestra y, mucho menos, se sistematiza.

Igualmente, sostiene Delgado (2012) que, la realidad social venezolana requiere de una nueva perspectiva curricular que ubique a la práctica profesional como un Formación de la Licenciatura en Servicios Penitenciarios en la Universidad Nacional Experimental De La Seguridad De Venezuela.

Aunado a los argumentos anteriormente esgrimidos, Gómez Grillo (2006), afirma que "la Administración Penitenciaria escogerá cuidadosamente el personal de eje de formación inicial universitaria que atienda la construcción y reconstrucción del conocimiento a partir del estudio de problemas sociales, desde y en la práctica como verdadero espacio de aprendizaje $y$, coadyuve a la generación de posibles alternativas de solución a los problemas de la sociedad. En este orden de ideas, surge la inquietud de analizar la práctica profesional penitenciaria como eje transversal de la estructura curricular en el Programa Nacional de todos sus grados, puesto que de la integridad, humanidad, aptitud personal $\mathrm{y}$ capacidad profesional de este personal dependerá la buena dirección de los establecimientos penitenciarios". De acuerdo con los anteriores planteamientos se propone Analizar la Práctica Profesional Penitenciaria como eje transversal de la estructura curricular en el Programa Nacional de Formación de la Licenciatura en Servicios Penitenciarios en la Universidad Nacional Experimental de la Seguridad de Venezuela.

\section{MÉTODOS}

Dada la naturaleza del objeto de estudio en esta investigación, el diseño de investigación es no experimental, porque en ella no está presente la manipulación de variables por parte de los investigadores. La información fue recolectada por los especialistas. El tipo de investigación es de campo ya que, se fundamenta en la medición de variables mediante la aplicación de un instrumento a una muestra de la población para el estudio de una situación social o institucional, en este caso se trata de la analizar la unidad curricular Práctica Profesional Penitenciaria como eje transversal en el Programa Nacional de Formación de la Licenciatura en Servicios Penitenciarios en la Universidad Nacional Experimental de la Seguridad de Venezuela.

\section{DISCUSIÓN Y RESULTADOS}

Se observa los datos que arrojan el gráfico 1 y el cuadro 1 que el $50 \%$ de la población encuestada posee conocimiento sobre el propósito de las prácticas profesionales penitenciarias 
Cuadro 1. Propósito

\begin{tabular}{|c|c|c|c|c|c|c|}
\hline & $\begin{array}{c}\text { Total Acuerdo } \\
\text { (TA) }\end{array}$ & $\begin{array}{c}\text { De Acuerdo } \\
\text { (DA) }\end{array}$ & Indiferente (I) & Desacuerdo (D) & $\begin{array}{c}\text { Total } \\
\text { Desacuerdo } \\
\text { (TD) }\end{array}$ & Total \\
\hline $\begin{array}{c}\text { ¿Conoce cuál es el propósito } \\
\text { de las prácticas profesionales } \\
\text { penitenciarias? }\end{array}$ & $\mathbf{9 0}$ & $\mathbf{7 0}$ & $\mathbf{1 0}$ & $\mathbf{8}$ & $\mathbf{2}$ & 180 \\
\hline
\end{tabular}

Total Acuerdo (TA) $\square$ De Acuerdo (DA) $\square$ Indiferente $(\mathrm{I}) \square$ Desacuerdo (D) $\square$ Total Desacuerdo (TD)

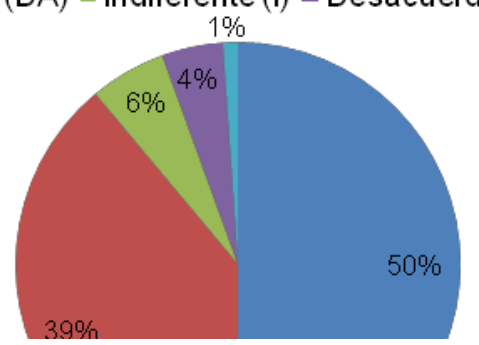

Grafico 1. Propósito de las prácticas profesionales penitenciarias. (Fuente: Los Investigadores, 2017)

Cuadro 2. Objetivo

\begin{tabular}{|c|c|c|c|c|c|c|}
\hline & $\begin{array}{c}\text { Total Acuerdo } \\
\text { (TA) }\end{array}$ & $\begin{array}{l}\text { De Acuerdo } \\
\text { (DA) }\end{array}$ & Indiferente (I) & Desacuerdo (D) & $\begin{array}{l}\text { Total } \\
\text { Desacuerdo } \\
\text { (TD) }\end{array}$ & Total \\
\hline $\begin{array}{c}\text { ¿Conoce cuál es el objetivo de } \\
\text { las prácticas profesionales } \\
\text { penitenciarias? }\end{array}$ & 90 & 70 & 10 & 8 & 2 & 180 \\
\hline
\end{tabular}

Total Acuerdo (TA) $\square$ De Acuerdo (DA) $\square$ Incliferente (I) $\square$ Desacuerdo(D) $\|$ Total Desacuerdo(TD)

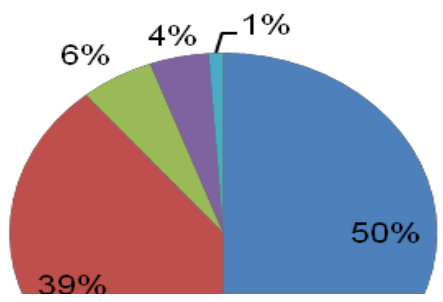

Gráfico 2. Objetivo de la prácticas profesionales penitenciarias. (Fuente: los investigadores, 2017)

Como se observa los datos que arrojan el sobre el propósito de las prácticas gráfico 2 y el cuadro 2 que el $50 \%$ de la profesionales penitenciarias.

muestra encuestada posee conocimiento 
Práctica profesional penitenciaria en la Universidad Nacional Experimental de la Seguridad de Venezuela durante el periodo 2015 - 2017

Como se observa, los datos que arrojan el gráfico 3 y el cuadro 3 que el $44 \%$ de la muestra encuestada posee conocimiento

sobre los destinatarios de las prácticas profesionales penitenciarias.

Cuadro 3. Destinatario

\begin{tabular}{|c|c|c|c|c|c|c|}
\hline & $\begin{array}{c}\text { Total Acuerdo } \\
\text { (TA) }\end{array}$ & $\begin{array}{c}\text { De Acuerdo } \\
\text { (DA) }\end{array}$ & Indiferente (I) & Desacuerdo (D) & $\begin{array}{c}\text { Total } \\
\text { Desacuerdo } \\
\text { (TD) }\end{array}$ & Total \\
\hline $\begin{array}{c}\text { ¿Conoce cuáles son los } \\
\text { destinatarios de las prácticas } \\
\text { profesionales penitenciarias? }\end{array}$ & $\mathbf{8 0}$ & $\mathbf{5 0}$ & $\mathbf{3 0}$ & $\mathbf{1 2}$ & $\mathbf{8}$ & $\mathbf{1 8 0}$ \\
\hline
\end{tabular}

Total Acuerdo (TA) $\square$ De Acuerdo(DA) $\square$ Indiferente (I) $\square$ Desacuerdo(D) $\quad$ Total Desacuerdo(TD)

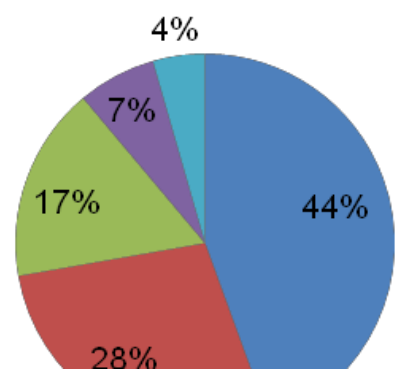

Gráfico 3. Destinatarios de las prácticas profesionales penitenciarias.

Los datos analizados, relacionados con los criterios teóricos - metodológicos necesarios implementados por el diseño de unidad curricular práctica profesional penitenciaria, para ello se estudió la opinión que posee la muestra encuestada sobre las categorías de análisis, estrategias instruccionales, estrategias metodológicas, estrategias de evaluación, duración, contenidos, modalidad de estudio.

En relación a ello, podemos señalar la aceptación por parte de la muestra encuestada entre el 30 y $60 \%$ de las estrategias instruccionales, estrategias metodológicas, duración, contenidos, y la modalidad de estudio, sin embargo, entre el 30 y $60 \%$ no está de acuerdo con las estrategias de evaluación, duración, contenidos, modalidad de estudio.

Como se observa los datos que arrojan el gráfico 4 y el cuadro 4 , existe en la muestra encuestada una posición controvertida el 39\% afirma estar totalmente de acuerdo con las estrategias instrucciones implementadas en las prácticas profesionales frente al 33\% señala estar en total desacuerdo.

Cuadro 4. Estrategias instruccionales

\begin{tabular}{|l|c|c|c|c|c|c|}
\hline & $\begin{array}{c}\text { Total Acuerdo } \\
\text { (TA) }\end{array}$ & $\begin{array}{c}\text { De Acuerdo } \\
\text { (DA) }\end{array}$ & Indiferente (I) & Desacuerdo (D) & $\begin{array}{c}\text { Total } \\
\text { Desacuerdo } \\
\text { (TD) }\end{array}$ & Total \\
\hline ¿Estrategias Instruccionales? & $\mathbf{7 0}$ & $\mathbf{3 0}$ & $\mathbf{0}$ & $\mathbf{2 0}$ & $\mathbf{6 0}$ & $\mathbf{1 8 0}$ \\
\hline
\end{tabular}


Total Acuerdo (TA) $\quad$ DeAcuerdo(DA) $\quad$ Indiferente (I) $\quad$ Desacuerdo(D) $\quad$ Total Desacuerdo(TD)

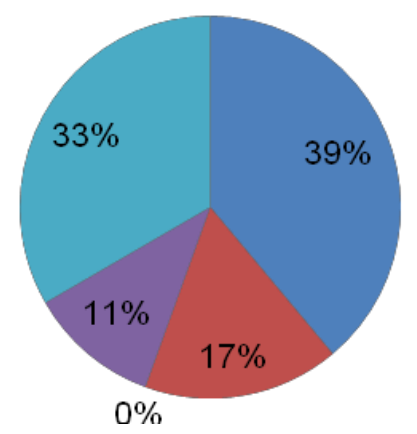

Gráficos 4. Estrategias instruccionales de las prácticas profesionales penitenciaria.

Como se observa los datos que arrojan el gráfico $5 \mathrm{y}$ el cuadro 5 existe en la muestra encuestada una posición controvertida el 39\% afirma estar totalmente de acuerdo con las estrategias metodológicas implementadas en las prácticas profesionales frente al 33\% señala estar en total desacuerdo.

Cuadro 5. Estrategias metodológicas.

\begin{tabular}{|l|c|c|c|c|c|c|}
\hline & $\begin{array}{c}\text { Total Acuerdo } \\
\text { (TA) }\end{array}$ & $\begin{array}{c}\text { De Acuerdo } \\
\text { (DA) }\end{array}$ & Indiferente (I) & Desacuerdo (D) & $\begin{array}{c}\text { Total } \\
\text { Desacuerdo } \\
\text { (TD) }\end{array}$ & Total \\
\hline ¿Estrategias Metodológicas? & $\mathbf{7 0}$ & $\mathbf{3 0}$ & $\mathbf{0}$ & $\mathbf{2 0}$ & $\mathbf{6 0}$ & $\mathbf{1 8 0}$ \\
\hline
\end{tabular}

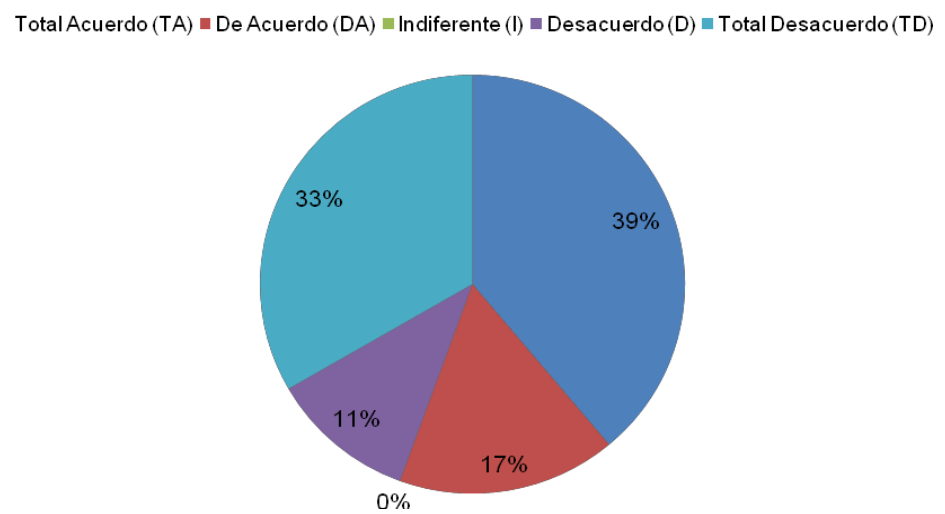

Gráfico 5. Estrategias metodológicas de las prácticas profesionales penitenciarias

Como se observa los datos que arrojan el gráfico 6 y el cuadro 6 la muestra encuestada plantea que el $39 \%$ afirma estar totalmente en desacuerdo con las estrategias de evaluación implementadas en las prácticas profesionales frente al 33\% señala estar en total acuerdo. 
Práctica profesional penitenciaria en la Universidad Nacional Experimental de la Seguridad de Venezuela durante el periodo 2015 - 2017

Cuadro 6. Estrategias de Evaluación

\begin{tabular}{|l|c|c|c|c|c|c|}
\hline & $\begin{array}{c}\text { Total Acuerdo } \\
\text { (TA) }\end{array}$ & $\begin{array}{c}\text { De Acuerdo } \\
\text { (DA) }\end{array}$ & Indiferente (I) & Desacuerdo (D) & $\begin{array}{c}\text { Total } \\
\text { Desacuerdo } \\
\text { (TD) }\end{array}$ & Total \\
\hline ¿Estrategias de Evaluación? & $\mathbf{6 0}$ & $\mathbf{3 0}$ & $\mathbf{0}$ & $\mathbf{2 0}$ & $\mathbf{7 0}$ & $\mathbf{1 8 0}$ \\
\hline
\end{tabular}

Total Acuerdo (TA) $\quad$ DeAcuerdo (DA) $\quad$ Indiferente (I) $\quad$ Desacuerdo(D) $\quad$ Total Desacuerdo(TD)

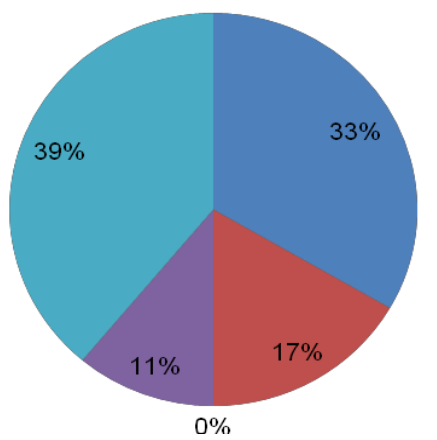

Gráfico 6. Estrategias de evaluación de las prácticas profesionales penitenciarias

Como se observa los datos que arrojan el gráfico 7 y el cuadro 7 la muestra encuestada plantea que el $56 \%$ afirma estar totalmente de acuerdo con los contenido programáticos de la unidad curricular Prácticas Profesionales Penitenciarias.

Cuadro 7. Contenidos programáticos

\begin{tabular}{|l|c|c|c|c|c|c|}
\hline & $\begin{array}{c}\text { Total Acuerdo } \\
\text { (TA) }\end{array}$ & $\begin{array}{c}\text { De Acuerdo } \\
\text { (DA) }\end{array}$ & Indiferente (I) & Desacuerdo (D) & $\begin{array}{c}\text { Total } \\
\text { Desacuerdo } \\
\text { (TD) }\end{array}$ & $\begin{array}{c}\text { Total } \\
\text { ¿Contenidos Programáticos? }\end{array}$ \\
\hline
\end{tabular}

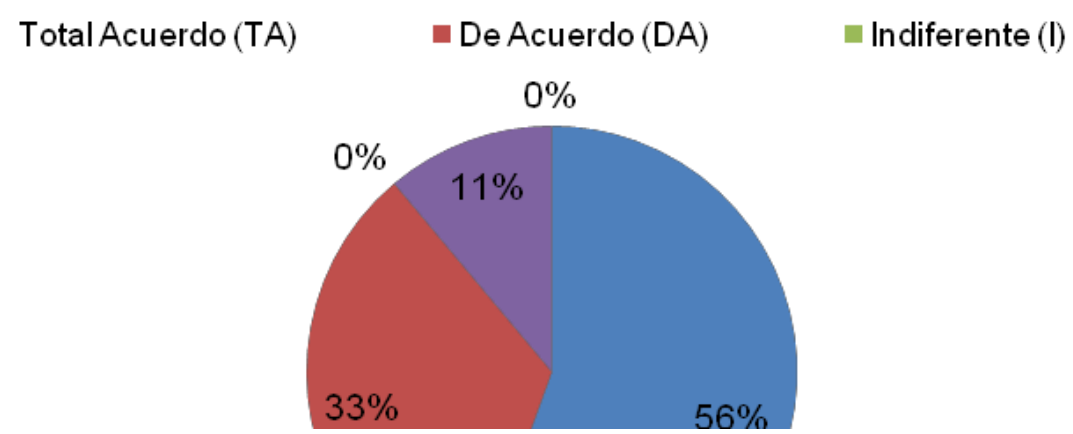

Gráfico 7. Contenidos programáticos de las prácticas profesionales penitenciario

Como se observa los datos que arrojan en el cuadro 8 la muestra encuestada plantea que el $56 \%$ afirma estar totalmente de acuerdo con duración de las prácticas profesionales penitenciarias. 
Cuadro 8. Duración

\begin{tabular}{|c|c|c|c|c|c|c|}
\hline & $\begin{array}{l}\text { Total Acuerdo } \\
\text { (TA) }\end{array}$ & $\begin{array}{l}\text { De Acuerdo } \\
\text { (DA) }\end{array}$ & Indiferente (I) & Desacuerdo (D) & $\begin{array}{c}\text { Total } \\
\text { Desacuerdo } \\
\text { (TD) }\end{array}$ & Total \\
\hline ¿Duración? & 100 & 60 & 0 & 20 & 0 & 180 \\
\hline
\end{tabular}

Como se observa los datos que arrojan el gráfico $8 \mathrm{y}$ el cuadro 9 . La muestra encuestada plantea que el $56 \%$ afirma estar totalmente de acuerdo con modalidad de estudio de las prácticas profesionales penitenciarias.

Total Acuerdo (TA) $\square$ De Acuerdo (DA) $\square$ Indiferente (I) $\square$ Desacuerdo (D) $\square$ Total Desacuerdo (TD)

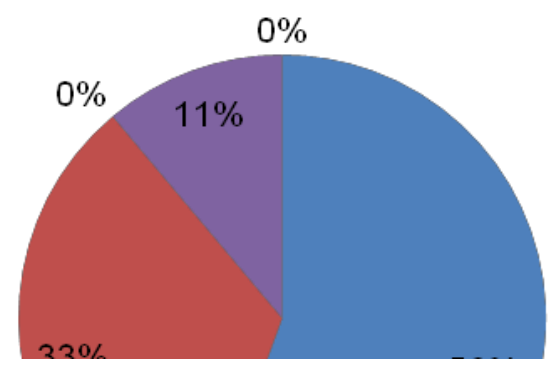

Gráfico 8. Modalidad de estudio del prácticas profesionales penitenciarias

El desarrollo de cualquier de las actividades planificadas el ámbito penitenciario es necesario que los responsables posean las competencias necesarias para el desempeño de su rol como servidor penitenciario, ya que se trata de una población muy sensible que se encuentra cumpliendo una pena en la mayoría de los caos privativa de libertad. En este contexto, las prácticas profesionales penitenciarias tienen un significativo impacto en virtud que garantiza la puesta en ejercicio del conocimiento académico y disminuye los riesgos del aprendizaje sobre la marcha, ya que, el órgano con competencia en servicio penitenciario no cuenta con un centro de entrenamiento y formación profesional para el mejoramiento continuo del equipo multidisciplinario.

En cuanto al, impacto de las actividades realizadas durante las prácticas en el alcance de las competencias de los profesionales en servicio penitenciario, como se observa los datos que arrojan el gráfico 9 y el cuadro 10 existe en la muestra encuestada una posición controvertida el $61 \%$ afirma estar totalmente de acuerdo frente al 39\% señala estar en total desacuerdo. Sin embargo, la tendencia es hacia el reconocimiento del impacto de estas actividades en la reinserción social del privado de libertad.

Cuadro 9. Modalidad de estudio

\begin{tabular}{|c|c|c|c|c|c|c|}
\hline & $\begin{array}{c}\text { Total Acuerdo } \\
\text { (TA) }\end{array}$ & $\begin{array}{c}\text { De Acuerdo } \\
\text { (DA) }\end{array}$ & Indiferente (I) & Desacuerdo (D) & $\begin{array}{c}\text { Total } \\
\text { Desacuerdo } \\
\text { (TD) }\end{array}$ & $\begin{array}{c}\text { Total } \\
\text { ¿Modalidad de Estudio? }\end{array}$ \\
\hline
\end{tabular}


Práctica profesional penitenciaria en la Universidad Nacional Experimental de la Seguridad de Venezuela durante el periodo 2015 - 2017

Total Acuerdo (TA) $\quad$ DeAcuerdo(DA) $\quad$ Indiferente (I) $\quad$ Desacuerdo(D) $\quad$ Total Desacuerdo(TD)

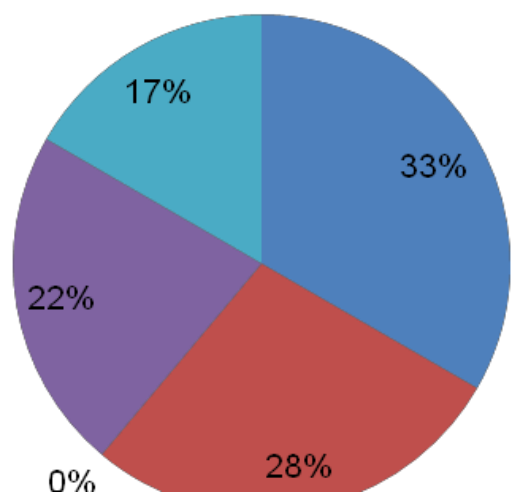

Gráfico 9. Actividades de las prácticas profesionales penitenciarias

Cuadro 10. Actividades acordes con las competencias a alcanzar

\begin{tabular}{|c|c|c|c|c|c|c|}
\hline & $\begin{array}{c}\text { Total Acuerdo } \\
\text { (TA) }\end{array}$ & $\begin{array}{c}\text { De Acuerdo } \\
\text { (DA) }\end{array}$ & Indiferente (I) & Desacuerdo (D) & $\begin{array}{c}\text { Total } \\
\text { Desacuerdo } \\
\text { (TD) }\end{array}$ & Total \\
\hline $\begin{array}{c}\text { ¿Cómo docente y trabajador } \\
\text { penitenciario que las actividades } \\
\text { que se realizan están acordes } \\
\text { con las competencias que debe } \\
\text { alcanzar el discente con la } \\
\text { unidad curricular práctica } \\
\text { profesional? }\end{array}$ & $\mathbf{6 0}$ & $\mathbf{5 0}$ & $\mathbf{0}$ & $\mathbf{4 0}$ & $\mathbf{3 0}$ & $\mathbf{1 8 0}$ \\
\hline
\end{tabular}

Como se observa los datos que arrojan el gráfico $10 \mathrm{y}$ el cuadro 11 existe en la muestra encuestada una posición controvertida el 44\% afirma estar totalmente de acuerdo que las actividades se realizan en su totalidad, frente al $66 \%$ señala estar en total desacuerdo.
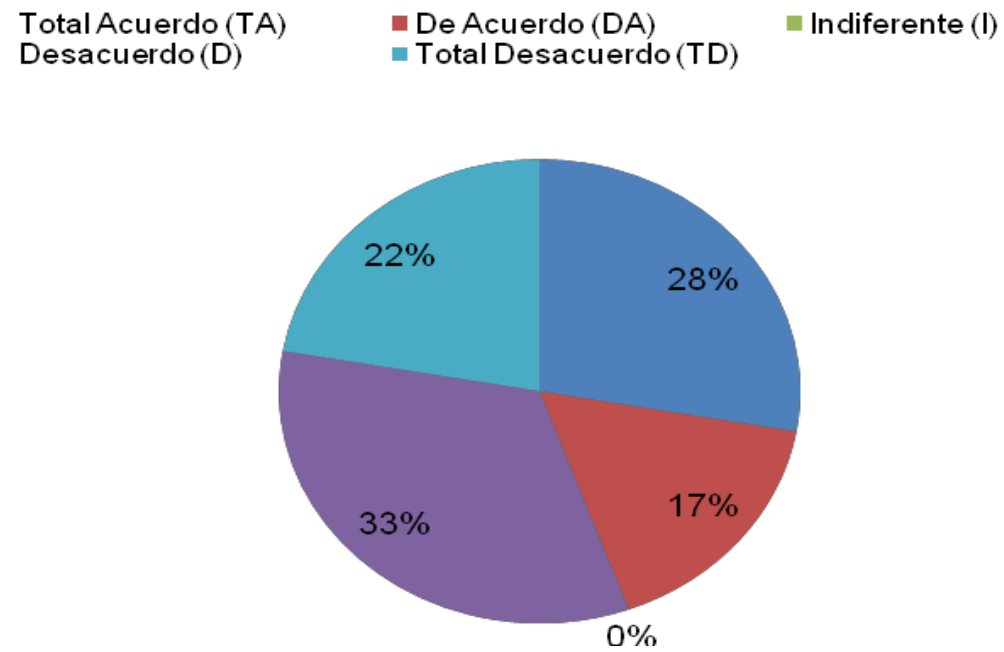

Gráfico 10. Cantidad de actividades de las prácticas profesionales penitenciarias 
Cuadro 11. Se realizan las actividades en su totalidad

\begin{tabular}{|l|c|c|c|c|c|c|}
\hline & $\begin{array}{c}\text { Total Acuerdo } \\
\text { (TA) }\end{array}$ & $\begin{array}{c}\text { De Acuerdo } \\
\text { (DA) }\end{array}$ & Indiferente (I) & Desacuerdo (D) & $\begin{array}{c}\text { Total } \\
\text { Desacuerdo } \\
\text { (TD) }\end{array}$ & Total \\
\hline $\begin{array}{c}\text { ¿Considera que las Actividades } \\
\text { se realizan en su totalidadl? }\end{array}$ & $\mathbf{5 0}$ & $\mathbf{3 0}$ & $\mathbf{0}$ & $\mathbf{6 0}$ & $\mathbf{4 0}$ & $\mathbf{1 8 0}$ \\
\hline
\end{tabular}

Como se observa en los datos que arrojan el gráfico 11 y el cuadro 12 existe en la muestra encuestada una posición controvertida el $44 \%$ afirma estar totalmente de acuerdo que existe articulación entre las instituciones que participan en las prácticas profesionales frente al $66 \%$ señala estar en total desacuerdo.

\section{Total Acuerdo (TA) Desacuerdo(D)}
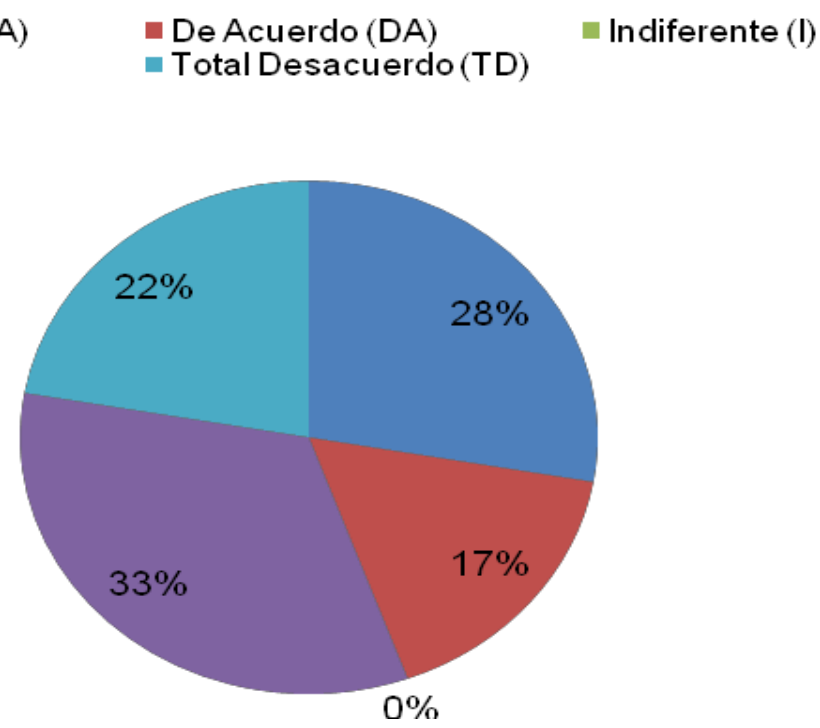

Gráfico 11. Articulación entre las instituciones que participan en las prácticas profesionales penitenciarias

\section{Cuadro 12. Articulación}

\begin{tabular}{|c|c|c|c|c|c|c|}
\hline & $\begin{array}{c}\text { Total Acuerdo } \\
\text { (TA) }\end{array}$ & $\begin{array}{c}\text { De Acuerdo } \\
\text { (DA) }\end{array}$ & Indiferente (I) & $\begin{array}{c}\text { Total } \\
\text { Desacuerdo (D) }\end{array}$ & $\begin{array}{c}\text { Desacuerdo } \\
\text { (TD) }\end{array}$ & Total \\
\hline $\begin{array}{c}\text { ¿Considera que existe } \\
\text { articulación entre las } \\
\text { instituciones que participan en } \\
\text { las prácticas profesionales? }\end{array}$ & $\mathbf{5 0}$ & $\mathbf{3 0}$ & $\mathbf{0}$ & $\mathbf{6 0}$ & $\mathbf{4 0}$ & $\mathbf{1 8 0}$ \\
\hline
\end{tabular}

Como se observa en los datos que arrojan el gráfico 12 y el cuadro 13 existe en la muestra encuestada una posición consolidada sobre la independencia de la unidad curricular
Práctica Profesional Penitenciaria el 83\% afirma estar totalmente de acuerdo frente al $27 \%$ señala estar en total desacuerdo. 
Gráfico 12. Articulación entre las instituciones que participan en las prácticas profesionales penitenciarias

Total Acuerdo (TA) $\square$ De Acuerdo(DA) $\square$ Indiferente (I) $\square$ Desacuerdo(D) $\square$ Total Desacuerdo(TD)

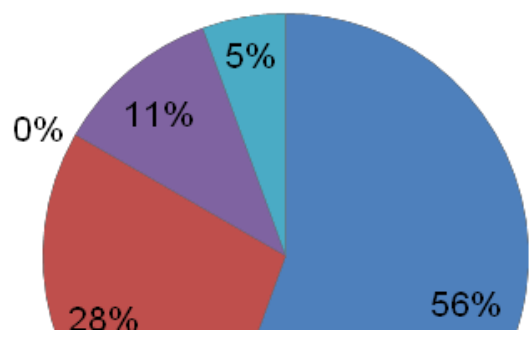

Cuadro 13. Independencia de la unidad curricular

\begin{tabular}{|c|c|c|c|c|c|c|}
\hline & $\begin{array}{c}\text { Total Acuerdo } \\
\text { (TA) }\end{array}$ & $\begin{array}{c}\text { De Acuerdo } \\
\text { (DA) }\end{array}$ & Indiferente (I) & Desacuerdo (D) & $\begin{array}{c}\text { Total } \\
\text { Desacuerdo } \\
\text { (TD) }\end{array}$ & Total \\
\hline $\begin{array}{c}\text { ¿Considera que la unidad } \\
\text { curricular Practica Profesional } \\
\text { debe ser un departamento } \\
\text { independiente de la unidad } \\
\text { curricular innovación e } \\
\text { investigación? }\end{array}$ & 100 & 50 & 0 & 20 & 10 & 180 \\
\hline
\end{tabular}

CONCLUSIONES

Una vez analizados los resultados obtenidos se hace evidente como resultado del diagnóstico que la muestra encuestada posee conocimiento sobre las categorías de análisis: propósito, objetivo y destinatarios, entre conclusiones se puede afirmar que el $50 \%$ y $40 \%$ de la muestra que posee conocimiento sobre el propósito, objetivo y destinatarios es decir sobre los mecanismos empleados para el desarrollo de las prácticas profesionales. Lo cual constituye una fortaleza ya que, la muestra está conformada por docentes y profesionales del área penitenciaria.

Existe aceptación por parte de la muestra encuestada entre el 30 y $60 \%$ de las estrategias instruccionales, estrategias metodológicas, duración, contenidos, y la modalidad de estudio, sin embargo, entre el 30 y $60 \%$ no está de acuerdo con las estrategias de evaluación, duración, contenidos, modalidad de estudio.

Como se observa los datos arrojan que existe en la muestra encuestada una posición controvertida el 61\% afirma estar totalmente de acuerdo frente al 39\% señala estar en total desacuerdo. Sin embargo, la tendencia es hacia el reconocimiento del impacto de estas actividades en pro de la reinserción de los privados de libertad ya que en la medida que la formación teórica practica del Licenciado en Servicios Penitenciarios brinde respuesta a las exigencias del entorno laboral donde se desarrolla. Sin embargo, existe una tendencia hacia la necesidad de transformar la unidad curricular Practica Profesional Penitenciaria en una actividad independiente de la unidad curricular Innovación e Investigación.

Una vez analizados los resultados obtenidos se hace evidente las la importancia de las prácticas profesionales en el desempeño profesional que deben poseer los 
Licenciados en Servicios Penitenciarios de la Universidad Nacional Experimental de la Seguridad, con propósito de optimizar su desempeño en el rol de defensoras y garantes de los derechos en un ámbito tan especial como es el penitenciario.

\section{REFERENCIAS}

Asamblea Constituyente (1999). Constitución de la República Bolivariana de Venezuela. (Gaceta Oficial № 5453 extraordinario) publicada el 24 de marzo de 2.000 .

Ley de Régimen Penitenciario. (2000). Gaceta Oficial de la República Bolivariana de Venezuela, № 36.975, 19-06-2000.

Asamblea Nacional. (2001). Ley de Reforma Parcial del Régimen Penitenciario Venezolano.

Casal, J. (2012). Los Derechos Humanos y su Protección. Caracas: Publicaciones UCAB.

Delgado, R. (2012) La práctica profesional como espacio de aprendizaje. Una perspectiva desde el enfoque de eje de la formación inicial universitaria. En:

Revista

Universitaria Arbitrada de Investigación y Diálogo Académico. 8, 2. Venezuela: Universidad

Pedagógica Experimental Libertador.

Kalinsky, B. (2012). El agente penitenciario: la cárcel como ámbito laboral. Runa, 28, 4357. Recuperado en 25 de junio de 2017, de http://www.scielo.org.ar/scielo.php?scr ipt=sci_arttext\&pid=S1851-

96282007000100003\&lng=es\&tlng=es 